\title{
Assessing the effectiveness of intervention strategies to address home injuries among children in Malaysia: a cluster randomized trial evaluation of evidence
}

\begin{abstract}
Background Malaysia is facing an increasing burden of childhood injuries, not unlike many other low- and middle-income countries (LMICs). Across the globe, more than 95 percent of total child injury mortality occurs in LMICs. A considerable proportion of these injuries occur in and around the home. Effective approaches to prevention include home safety education, parenting education and home modification. Through this study, we aim to evaluate the effectiveness of two intervention strategies in reducing in-home hazards for unintentional injuries among children. Methods We conducted a prospective cluster randomized controlled trial (cRCT) to compare the effectiveness of an in-home safety tutorial program and an educational pamphlet intervention in reducing safety hazards to child injuries. The study randomized 39 clusters to two study arms (home safety tutorial vs educational pamphlet), with around 30 households per cluster. Our exposure and outcome were assessed using a household survey instrument with self-reported and observer-reported components. Results Our initial analysis is a comparison of overall safety scores across the baseline arm that found a mean safety score of 30 of a maximum possible 45 (translating to a mean percentage of $66.7 \%$ ), with improvement on average in both intervention arms. The overall safety score mean for educational pamphlets was 32.3 with a mean percentage of $71.7 \%$, and the corresponding numbers for the in-home tutorial were 31.8 and $71 \%$. Discussion This study compares a labor-intensive and 'active' intervention, the in-home safety tutorial, with a more passive intervention through handing out educational pamphlets. In our preliminary findings from the cRCT, we see an improvement in the mean safety scores between baseline and follow-up measurement in both intervention arms; between the two intervention arms, the safety scores are comparable. We believe that educational pamphlets can be an effective intervention to reduce child injuries, with particular relevance to resource constrained settings.
\end{abstract}

